\title{
Au sujet de la variation du coefficient de réaction du sol en fonction de l'inertie des écrans de soutènement
}

On peut observer, à travers les récents articles consacrés, dans la Revue Française de Géotechnique, au coefficient de réaction du sol vis-à-vis des écrans de soutènement, un certain consensus sur le fait que ce coefficient, $k$, dépend du produit d'inertie, EI, de l'écran.

En revanche, une divergence importante subsiste dans l'apprèciation du sens de variation de k en fonction de EI.

En effet, nous avons relevé, dans les numéros 66 farticle de A. Monnet: «Module de réaction, coefficient de décompression. au sujet des paramètres utilisês dans la méthode de calcul élastoplastique des soutènements )), 68 (discussion au sujet de l'article de A. Monnet) et 72 (article de A. Dhoulb: "Méthode des coefficients de réaction appliquée en France pour les calculs des soutènements: influence de la variation des paramètres $\nu$ ), l'indication d'une variation croissante de $k$ en fonction de EI.

Par contre, nous avons, dans le numéro 71 (article de P. Schmitt: « Méthode empirique d'évaluation du coefficient de réaction du sol vis-à-vis des ouvrages de soutènement souples »), mis en évidence une variation nettement décroissante.

Il apparait donc une divergence profonde entre ces différents points de vue, divergence sur laquelle nous voudrions essayer d'apporter quelques éléments d'explication.

Tout d'abord, il nous semble que le fait, indiscutable, que le déplacement d'un écran rigide soit plus faible que celui d'un écran déformable, toutes choses égales par ailleurs, ne permet pas de conduire à une plus forte valeur de $k$.

En effet, l'augmentation de EI est par elle-méme une cause essentielle de la limitation du déplacement, et ce indépendamment de la variation de $k$.

Pour pouvoir conclure à une augmentation de $k$, il faucirait avoír observé, pour un écran d'inertie croissante, une diminution du déplacement, $\Delta y$, sans diminution notable, voire avec augmentation de la variation de pression associée, $\Delta p$.

Tel parait ètre effectivement le cas si l'on s'intéresse au comportement élastique du sol en poussée. état dans lequel la pression, $p$ augmente quand $\Delta y$ diminue, donc quand l'inertie de l'écran augmente.

Cependant, bien que la pression p soit plus importante pour un écran rigide que pour un écran souple, on ne peut en conclure que la variation de pression élastique, $\Delta \mathrm{p}$, associée au déplacement, $\Delta \mathrm{y}$, est elle-mème plus importante.

On peut, certes, observer que $\Delta \mathrm{p}$ augmente globalement quand $\Delta y$ diminue, dans la mesure où les petites déformations intéressent exclusivement le domaine élastique du sol, et sont donc décrites par le coefficient de réaction élastique $\mathrm{k}=\Delta \mathrm{p} / \Delta \mathrm{y}$, tandis que les déformations plus importantes inté- ressent rapidement le domaine plastique, décrit par le coefficient de poussée active, et qui donne lieu à des déplacements plus élevés: on peut en déduire que le coefficient de réaction $\alpha$ apparent $»$ du sol décomprimé est plus élevé pour un écran rigide que pour un écran souple, mais on ne peut rien en déduire vis-à-vis du coefficient de réaction èlastique proprement dit.

On peut par contre noter que, cornme l'indique A. Dhouib, la prise en compte d'une loi non linéaire traduirait mieux le comportement du sol : mais il faudrait alors tenir compte d'une formulation du type $\mathrm{k}=\mathrm{f}(\mathrm{y})$, et non $\mathrm{k}=\mathrm{f}(\mathrm{E})$, dans la mesure où le déplacement de l'écran est loin d'être uniquement fonction de son inertie.

En pratique, le domaine élastique du sol en poussée est relativement limité, ce qui explique que l'on se contente en général. forfaitairement, d'assimiler le coefficient de réaction en décompression au coefficient de réaction en compression, plus accessible à l'expérience et plus intéressant pour le concepteur: en effet, le domaine élastique du sol en butée est considérablement plus étendu qu'en poussée, et c'est donc le coefficient de réaction du sol comprimé qui constitue, dans le domaine élastique, le paramètre déterminant vis-à-vis du comportement de l'écran.

Or dans le cas de la butée, un effort exercé sur le sol par l'intermédiaire d'un écran rigide sera mieux réparti que par un écran souple, et donnera donc naissance à des contraintes plus faible.

Cette plus grande répartition des contraintes par un écran rigide se traduit par une plus grande valeur du paramètre dimensionnel, "a », hauteur sur laquelle le terrain se trouve réellement sollicité par l'écran, ce qui, compte tenu de la théorie du bulbe des isocontraintes de Terzaghi selon laquelle le coefficient de réaction est inversement proportionnel à ( a $)$, amène à la conclusion, corroborée par les muitiples expérimentations dont nous avons fait état. que $k$ varie en raison inverse de El.

A notre connaissance, trois formulations au moins permettent aujourd'hui de tenir compte de cette variation

- Vesic, cité par Simon dans le $n^{\circ} 71$ de la RFG, dans le cas d'une poutre de largeur b et de grande longueur, sollicitée ponctuellement:

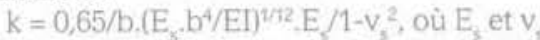
sont les caractéristiques élastiques dú sol.

- Balay et Gigan, dans le cas d'une mise en tension de tirant:

$k=r \cdot s \cdot 4 \cdot E_{M} /\left(E l / E_{M}\right)^{1 / 3}$, où $E_{M}$ est le module pressiométrique, $\mathrm{r}$ et $\mathrm{s}$ deux coefficients adimensionnels.

- Schmitt, dans le cas général d'un écran continu (formulation « moyenne s) intégrant, sur une base empirique, les différents modes de sollicitations classiques):

$\mathrm{k}=2,1 .\left(\mathrm{E}_{4} / \alpha\right)^{43} / \mathrm{El}^{1 / 3}$, où $\alpha$ est le "coefficient de structure n du sol. 
En revanche, la formule proposée par A. Monnet présente, bien qu'également destinée à décrire la réaction du sol comprimé la tendance inverse, puisque El y figure au numérateur et non au dénominateur:

$k=\left(20 . E I \cdot\left(K_{p} \cdot \gamma \cdot\left(1-K_{o} / K_{p}\right) / d r_{0}\right)^{4}\right)^{1 / 5}+$ $A \cdot C^{\prime} \cdot \operatorname{th}\left(C^{\prime} / C_{0}\right) / d r_{0}$

En réalité, cette divergence peut s'expliquer si l'on observe que la formule de Monnet, tout comme l'abaque de Chadeisson qu'elle cherche à généraliser, approche le coefficient de réaction élastique à partir de la prise en compte de l'équilibre plastique du sol, lui-mème déterminé à partir des coefficients de butée classiques, et de l'ordre de grandeur, supposé connu, du déplacement, $\mathrm{dr}$, nécessaire à l'obtention de cet équilibre-limité.

Il est clair que ces approches sont intéressantes dans la mesure où elles permettent d'obtenir des ordres de grandeur gènéralement plausibles, largement validés par l'usage dans le cas de l'abaque de Chadeisson, et corroborés par l'expérience dans le cas de parois moulées d'épaisseurs courantes et de terrains suffisamment compacts.

Elles ne peuvent par contre prétendre décrire de façon précise l'influence du mode de déformation de l'écran, dans la mesure oú les déformations ne sont appro- chées qu'à partir d'hypothèses forfaitaires, à la fois sur leur valeur moyenne et leur distribution, et que la validité des formulations qui en découlent dépend exclusivement de la validité de ces hypothèses,

La difficulté inhérente à ces approches est encore exacerbée du fait que le domaine plastique n'est pas atteint simultanément sur toute la hauteur sollicitée, et que les déplacements en jeu intègrent donc à la fois des déplacements élastiques, approchés par la théorie du coefficient de réaction, et des déplacements plastiques indéterminés, évalués forfaitairement, et en pratique assimilés aux précédents, ce quí peut expliquer l'obtention d'un résultat paradoxal du point de vue des déformations élastiques.

En définitive, il nous semble que les divergences signalées ne sont qu'apparentes, puisque les problèmes traités sont en toute riqueur de natures différentes massif élastique comprimé ou décomprimé, et équilibre-limite du sol

Dans le cas de la butée élastique, les différentes formulations existentes permettent bien, compte tenu de leurs supports théoriques et expérimentaux, de conclure à une variation décroissante du coefficient de réaction du sol lorsque l'inertie de l'écran augmente. 